\title{
Photoexcitation Dynamics of S-Nitrosoglutathione Probed by Femtosecond Mid-IR Spectroscopy
}

\author{
Jaeheung Park, Seongheun Kim, and Manho Lim* \\ Department of Chemistry and Chemistry Institute for Functional Materials, Pusan National University, Busan 609-735, Korea \\ *E-mail:mhlim@pusan.ac.kr \\ Received March 28, 2005
}

Key Words : Nitric oxide (NO), S-Nitrosoglutathione (GSNO), Photodecomposition, Femtosecond mid-IR spectroscopy

Nitric oxide (NO) has been found to perform a number of physiological functions such as smooth muscle relaxation, platelet inhibition, neurotransmission, immune regulation, and penile erection. ${ }^{1}$ S-nitrosothiols (RSNOs), which are less reactive relative to the free radical form of $\mathrm{NO}$ and readily formed in vivo as part of the metabolism of NO, play significant roles in the uptake, intracellular trafficking and release of NO within the body ${ }^{1-3}$ Because NO is also found to function as macrophage-mediated cytotoxic agent ${ }^{4}$ and RSNO undergoes photodecomposition by UV or visible light, ${ }^{5}$ there is a growing interest in RSNO as NO carriers to target areas (e.g., tumors) where NO could act as a photoactivated cytotoxic agent. ${ }^{3,5,6}$ However due to the facile $\mathrm{S}-\mathrm{N}$ bond decomposition of RSNO by heat, light, or $\mathrm{Cu}^{+}$ ions, experimental studies on RSNOs are often quite difficult. ${ }^{3,7}$ Computationally the $6-311+\mathrm{G}(2 \mathrm{df}, \mathrm{p})$ or larger basis set was found to be needed to obtain reliable structure. ${ }^{8}$ Consequently, our current level of understanding of many physical and spectroscopic properties of RSNOs is not satisfactory.

Glutathione (GSH), the most abundant nonprotein thiol both in plasma and inside all cells, is known to form an Snitrosothiol, GSNO in vivo. ${ }^{9}$ GSNO is stable to isolation and storage in the dark. ${ }^{5}$ The stability of GSNO, a primary RSNO, is unusual knowing that substitution at sulfur is a primary factor determining the stabilities of RSNOs: it was reported that most of primary and secondary RSNOs have half-lives of seconds to minutes and tertiary ones are stable for long-term storage. ${ }^{10}$ It has been studied as both naturally occurring NO-carrier and a model system for albumin, the principal bloodstream host. ${ }^{11}$ Use of GSNO as a photochemotherapeutic agent has been explored, too. ${ }^{5}$ Here we have used femtosecond vibrational spectroscopy to probe details of photoexcitation dynamics and mechanism of S-N bond cleavage of GSNO by near-UV. We have found an excited electronic state of GSNO that might play a critical role in photolysis of S-N bond of GSNO. The stretching frequency of $\mathrm{NO}$ in the new electronic state is very close to that of the free radical form of NO, suggesting that electronic structure of bound $\mathrm{NO}$ in the new electronic state is similar to that of the free radical form.

The spectrometer used to collect the time-resolved spectra, described in detail elsewhere, ${ }^{12,13}$ consists of two optical parametric amplifiers (OPAs) pumped by a Ti:sapphire amplifier. They are used to generate a $320 \mathrm{~nm}$ pump pulse and a mid-IR probe pulse. Frequency quadrupled signal from one OPA generates the near-UV light. Mid-IR probe pulses are generated by difference frequency mixing of the signal and idler pulses of the other OPA. The broadband transmitted IR pulse is detected with a 64-elements $\mathrm{N}_{2}(l)$ cooled $\mathrm{HgCdTe}$ array detector which is mounted in the focal plane of a $320 \mathrm{~mm}$ monochromator with a $120 \mathrm{l} / \mathrm{mm}$ grating, resulting in a spectral resolution of $c a .1 .6 \mathrm{~cm}^{-1} /$ pixel at $1830 \mathrm{~cm}^{-1}$, and $1.1 \mathrm{~cm}^{-1} /$ pixel at $1520 \mathrm{~cm}^{-1}$. The spectra spanning $160 \mathrm{~cm}^{-1}\left(1520 \mathrm{~cm}^{-1}\right.$ region $)$ are a superposition of three 64-point spectra that overlap by a few elements. The polarization of the pump pulse was adjusted to recover the isotropic absorption spectra and anisotropy of the spectra. The instrument response function is typically $250 \mathrm{fs}$.

GSH and $\mathrm{NaNO}_{2}$ (both from Aldrich) were used to synthesize GSNO according to the method of Hart. ${ }^{14}$ A 50 $\mathrm{mM}$ GSNO in $\mathrm{D}_{2} \mathrm{O}$ was loaded in a gas-tight $50\left(1520 \mathrm{~cm}^{-1}\right.$ region) or $100-\mathrm{mm}$-pathlength $\left(1830 \mathrm{~cm}^{-1}\right.$ region $) \mathrm{CaF}_{2}$ flowing cell. During data collection the sample was flowed sufficiently fast so that each photolyzing laser pulse illuminated a fresh volume of the sample. The sample was prepared in $\mathrm{D}_{2} \mathrm{O}$ to isotopically shift the spectral region of interest to a region with greater IR transmission. The temperature of the sample cell was kept at $283 \pm 1 \mathrm{~K}$ to minimize thermal decomposition of sample. Throughout the experiments UV-Vis spectroscopy was used to ensure the integrity and concentration of sample.

Figure 1 shows representative time-resolved mid-IR absorption spectra after photoexcitation of GSNO in $\mathrm{D}_{2} \mathrm{O}$ at $283 \mathrm{~K}$. We probed spectral ranges from 2050 to $1350 \mathrm{~cm}^{-1}$. Spectral features larger than $0.02 \mathrm{mOD}$ are found only in the regions shown in Figure 1. The negative going features over the spectral range $1430-1630 \mathrm{~cm}^{-1}$ arise from the loss of the compound in the ground electronic state. They consist of two spectral features: one peaked near $1520 \mathrm{~cm}^{-1}$ and the other at $1476 \mathrm{~cm}^{-1}$. The feature near $1520 \mathrm{~cm}^{-1}$, exhibiting a ${ }^{15} \mathrm{NO}$ isotopic shift, corresponds to NO stretching mode in the ground electronic state. It consists of two spectral bands that represent two geometrical isomers arising from S-N double bond character. One band is peaked at $1549 \mathrm{~cm}^{-1}$ with $80 \mathrm{~cm}^{-1}$ full width at half maximum (FWHM) and the 

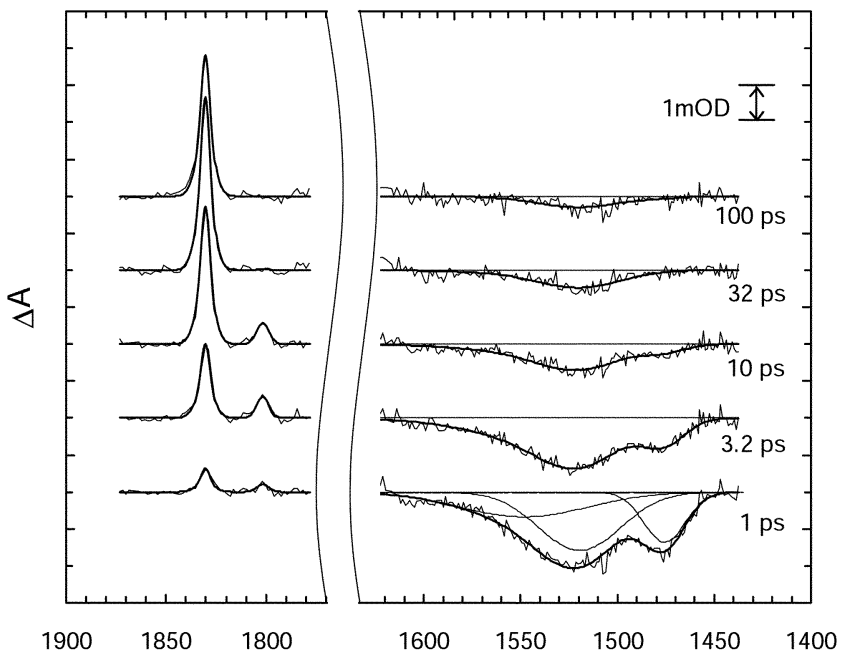

$\mathrm{E} / \mathrm{hc}\left(\mathrm{cm}^{-1}\right)$

Figure 1. Representative time-resolved vibrational spectra of $\mathrm{NO}$ after photoexcitation of GSNO in $\mathrm{D}_{2} \mathrm{O}$ at $283 \mathrm{~K}$. The negativegoing spectra (the thin solid lines) are fit to two features (the thick solid lines). One feature peaked near $1520 \mathrm{~cm}^{-1}$ is modeled as a sum of two Gaussians and the other feature at $1476 \mathrm{~cm}^{-1}$ a Gaussian. The two Gaussians for the feature near $1520 \mathrm{~cm}^{-1}$ correspond to two N-O stretching modes bound to GSNO in the ground electronic state. Two modes represent two geometrical isomers arising from S-N double bond character in S-nitrosothiols. The band at $1476 \mathrm{~cm}^{-1}$ is attributed to the ND bending mode the bleach of which is induced by photoexcitation. The spectrum at $1 \mathrm{ps}$ is decomposed to its component. The positive-going features are fit with a sum of two Gaussians and their red-shifted replicas. The red-shifted replicas represent vibrationally hot NO (see text). For clarity, the linear polynomial background has been subtracted from the measured spectra.

other one at $1520 \mathrm{~cm}^{-1}$ with $50 \mathrm{~cm}^{-1} \mathrm{FWHM}$. The bands at $1549 \mathrm{~cm}^{-1}$ and $1520 \mathrm{~cm}^{-1}$ correspond to the compound in anti and syn orientations, respectively. ${ }^{15}$ Assuming that the population of GSNO in each isomer is proportional to its integrated absorbance, the equilibrium population ratio, [syn]/[anti] is $c a .1 .9$ and free energy of GSNO in syn orientation is $c a .1 .6 \mathrm{~kJ} / \mathrm{mol}$ lower than that in anti orientation. It is consistent with the finding that syn orientation is favored in the primary RSNO. ${ }^{16}$ The bleach of two bands, indistinguishable in our $\mathrm{S} / \mathrm{N}$ ratio, recovers on two time constants, 4 and $120 \mathrm{ps}$, respectively. Photoexcitation dynamics and the S-N bond cleavage are likely independent of the isomeric form of the compound. The band at 1476 $\mathrm{cm}^{-1}$, independent of ${ }^{15} \mathrm{NO}$ isotopic substitution, can be attributed to the ND bending mode of the peptide ${ }^{17}$ and its bleach likely arises from photoinduced conformational change that influences $\mathrm{NO}$ bending mode. It recovers with 3 ps time constant.

The positive going features over the spectral range 1780$1870 \mathrm{~cm}^{-1}$ arise from the gain of NO population since both features show the ${ }^{15} \mathrm{NO}$ isotopic shift. While the band at $1830 \mathrm{~cm}^{-1}$ is attributed to the fundamental NO stretching mode, the one at $1802 \mathrm{~cm}^{-1}$ is attributed to the $\mathrm{v}=1 \rightarrow \mathrm{v}=2$ transition of NO (hot band) because it is the replica of the band at $1830 \mathrm{~cm}^{-1}$ and red-shifted by $28 \pm 1 \mathrm{~cm}^{-1}$, similar to

anharmonicity of NO in the gas phase, $27.94 \mathrm{~cm}^{-1} .^{18}$ The band at $1830 \mathrm{~cm}^{-1}$ was observed in the previous experiment and, based on the fact that the frequency is near that of the gas phase NO absorbing at $1876.09 \mathrm{~cm}^{-1},{ }^{18}$ assigned to photoreleased NO. ${ }^{19}$ However, we have found that the bands arise not from solvated "free NO" but from bound NO in a new electronic state (vide infra). While both bands rise with a 3.8 ps time constant, the band at $1830 \mathrm{~cm}^{-1}$ decays with a $180 \mathrm{ps}$ time constant and the one at $1802 \mathrm{~cm}^{-1}$ with $11 \mathrm{ps}$, the vibrational relaxation (VR) time of $\mathrm{NO}$ in the new state.

Free NO spectrum solvated in Ar matrix is found to be peaked at $1872 \mathrm{~cm}^{-1} \cdot{ }^{20}$ We have attempted to measure vibrational spectrum of free $\mathrm{NO}$ in $\mathrm{D}_{2} \mathrm{O}$ at ambient temperature, found no noticeable spectral features, and concluded that the integrated extinction coefficient of the spectrum for free $\mathrm{NO}$ in $\mathrm{D}_{2} \mathrm{O}$ at ambient temperature is $<200 \mathrm{M}^{-1} \mathrm{~cm}^{-2}$. At our best knowledge, vibrational spectrum of free $\mathrm{NO}$ in $\mathrm{D}_{2} \mathrm{O}$ at ambient temperature has not been characterized. The lack of spectral features of the radical in water may result from inhomogeneous broadening and low extinction coefficient of the spectrum as well as its high reactivity. Recently we have obtained vibrational spectrum of free $\mathrm{NO}$ within a protein cavity at $283 \mathrm{~K}$ by photolyzing NO-ligated myoglobin. ${ }^{21}$ It is peaked near $1870 \mathrm{~cm}^{-1}$ with $10-14 \mathrm{~cm}^{-1}$ FWHM. The integrated extinction coefficient is $c a .460 \mathrm{M}^{-1} \mathrm{~cm}^{-2}$. As summarized in Table 1, vibrational spectra of free $\mathrm{CO}$ in $\mathrm{D}_{2} \mathrm{O}$ as well as inside protein have been well characteriz$\mathrm{ed}^{22-24}$ Because the spectral characteristics of free $\mathrm{CO}$ and free $\mathrm{NO}$ in protein and in the gas phase are similar, we assumed that the vibrational spectrum of free $\mathrm{NO}$ solvated in $\mathrm{D}_{2} \mathrm{O}$ is similar to that of $\mathrm{CO}$. An estimated spectrum of free $\mathrm{NO}$ solvated in $\mathrm{D}_{2} \mathrm{O}$ may have the following characteristics: $\bar{v} \sim 1870 \mathrm{~cm}^{-1}$, FWHM $\sim 30 \mathrm{~cm}^{-1}, \int \varepsilon d \bar{v} \sim 200 \mathrm{M}^{-1} \mathrm{~cm}^{-2}$, and VR time is hundreds of picoseconds.

By comparing the estimated spectrum of free $\mathrm{NO}$ in $\mathrm{D}_{2} \mathrm{O}$ with the new band, we could conclude that the new band arises not from free NO but from bound NO in a new electronic state. The following is the noticeable characteristics suggesting that the band corresponds to bound NO. (1) VR time of $11 \mathrm{ps}$ is too short to be that of free NO. It is

Table 1. Spectral characteristics of vibrational absorption spectra of $\mathrm{CO}$ and $\mathrm{NO}$

\begin{tabular}{ccccc}
\hline & \multicolumn{2}{c}{${ }^{13} \mathrm{CO}$} & & $\mathrm{NO}$ \\
\cline { 2 - 3 } \cline { 5 - 5 } Environment & $\begin{array}{c}\text { in } \mathrm{D}_{2} \mathrm{O} \\
\text { near } 300 \mathrm{~K}^{22}\end{array}$ & $\begin{array}{c}\text { in a protein } \\
\text { internal cavity } \\
\text { at } 283 \mathrm{~K}^{23,24}\end{array}$ & & $\begin{array}{c}\text { in a protein } \\
\text { internal cavity } \\
\text { at } 283 \mathrm{~K}^{21}\end{array}$ \\
\hline $\bar{v}\left(\mathrm{~cm}^{-1}\right)^{a}$ & 2091 & 2080 & & 1870 \\
$\Delta\left(\mathrm{cm}^{-1}\right)^{b}$ & -5 & -16 & & -6 \\
$\mathrm{FWHM}\left(\mathrm{cm}^{-1}\right)$ & 27 & $7-14$ & & $10-14$ \\
$\int \varepsilon d \bar{\nu}\left(\mathrm{M}^{-1} \mathrm{~cm}^{-2}\right)^{c}$ & $\sim 400$ & $\sim 740$ & & $\sim 460$ \\
VR time & & $600 \mathrm{ps}$ & $\sim 200 \mathrm{ps}$ \\
\hline
\end{tabular}

${ }^{a}$ Band position of the respective transition. The medium values are taken for two bands in a protein internal cavity. ${ }^{b}$ Shift relative to band position in the gas phase $\left({ }^{13} \mathrm{CO} \rightarrow 2096.0 \mathrm{~cm}^{-1} ; \mathrm{NO} \rightarrow 1876.09 \mathrm{~cm}^{-1}\right)$. ${ }^{c}$ Integrated extinction coefficient of the narrow band. ${ }^{21}$ 
rather similar to the VR time of bound NO. VR times of NO bound to transition metals are likely tens of picoseconds. (2) Spectral bandwidth of $7 \mathrm{~cm}^{-1}$ is much narrower than estimated. It is even narrower than spectral bandwidth of NO bound to transition metal. ${ }^{13}$ (3) Spectral shift relative to band position in the gas phase, $46 \mathrm{~cm}^{-1}$ is much larger than estimated shift, $6 \mathrm{~cm}^{-1}$. (4) The appearance time of $3.8 \mathrm{ps}$ is too slow to be photogeneration time of $\mathrm{NO}$ or solvation time of photodissociated $\mathrm{NO}$ in $\mathrm{D}_{2} \mathrm{O}$. Photodissociation of $\mathrm{CH}_{3} \mathrm{SNO}$ by near UV is suggested to occur much faster than $100 \mathrm{fs}^{25}$ and solvent rearrangement of $\mathrm{D}_{2} \mathrm{O}$ occurs on the subpicosecond time scale. ${ }^{26}(5)$ Integrated extinction coefficient is estimated to be $11000 \mathrm{M}^{-1} \mathrm{~cm}^{-2}$, which is $30-55$ times larger than the estimated value. Interestingly integrated extinction coefficient of bound NO to heme is about 25 times larger than that of free $\mathrm{NO}^{27}$ (6) A preliminary anisotropy experiment shows that the anisotropy observed at time $t$ after pumping, $r(t)=0.13 \exp (-t / 2 \mathrm{ps})+0.2 \exp (-t / 70$ ps). Slow component of the anisotropy decay also confirms that the band arise from bound NO. Anisotropy of free NO would decay much faster. The anisotropy value, $r(0)=0.33$ reveals the geometrical relationship between the pumped and probed transition dipoles. Because experiment tends to underestimate due to some depolarization of the pump and probe pulses and partial saturation of the transition, the measured anisotropy is the lower limit. Thus the angle between the two transition dipoles is $<20^{\circ}$.

In conclusion we have found a new $\mathrm{NO}$ absorption band near that of free NO gas and assigned the band to NO bound to an excited electronic state. The fact that vibrational frequency of NO in the new electronic state is much closer to that of NO radical and very different from that in the ground electronic state suggests that the chemical nature of NO in the new state is closer to the free radical and very different from NO in the ground electronic state of GSNO. The transition dipole of the new NO absorption is close to collinear with that of near UV. This new band was not observed in photoexciation of SNAP (a tertiary RSNO). We speculate that the existence of the new state might contribute to the exceptional stability of GSNO compared with other primary RSNOs.

Acknowledgements. This work was supported by grants from the Korea Science and Engineering Foundation through CIMS (POSTECH).

\section{References}

1. Stamler, J. S.; Loscalzo, J. J. Cardiovas. Pharm. 1992, 20, S202-
S204.

2. (a) Stamler, J. S. Cell 1994, 78, 931-6 (b) Stamler, J. S.; Jaraki, O.; Osborne, J.; Simon, D. I.; Keaney, J.; Vita, J.; Singel, D.; Valeri, C. R.; Loscalzo, J. Proc. Natl. Acad. Sci. U.S.A. 1992, 89, 7674 7677.

3. Williams, D. L. H. Acc. Chem. Res. 1999, 32, 869-876.

4. Stuehr, D. J.; Nathan, C. F. J. Exp. Med. 1989, 169, 1543-1555.

5. Sexton, D. J.; Muruganandam, A.; McKenney, D. J.; Mutus, B Photochem. Photobiol. 1994, 59, 463-7.

6. (a) Singh, R. J.; Hogg, N.; Joseph, J.; Kalyanaraman, B. J. Biol. Chem. 1996, 271, 18596-18603 (b) Wood, P. D.; Mutus, B.; Redmond, R. W. Photochem. Photobiol. 1996, 64, 518524.

7. Lipton, A. J.; Johnson, M. A.; Macdonald, T.; Lieberman, M. W.; Gozal, D.; Gaston, B. Nature 2001, 413, 171-174.

8. (a) Arulsamy, N.; Bohle, D. S.; Butt, J. A.; Irvine, G. J.; Jordan, P. A.; Sagan, E., J. Am. Chem. Soc. 1999, 121, 7115-7123 (b) Baciu, C.; Gauld, J. W. J. Phys. Chem. A 2003, 107, 9946-9952.

9. Meister, A.; Anderson, M. E. Annu. Rev. Biochem. 1983, 52, 711760 .

10. Bartberger, M. D.; Mannion, J. D.; Powell, S. C.; Stamler, J. S.; Houk, K. N.; Toone, E. J. J. Am. Chem. Soc. 2001, 123, 8868-9.

11. Mohney, B. K.; Walker, G. C. J. Am. Chem. Soc. 1997, 119, $9311-$ 9312.

12. Kim, S.; Jin, G.; Lim, M. Bull. Korean Chem. Soc. 2003, 24, 14701474. (b) Kim, S.; Heo, J.; Lim, M. Bull. Korean Chem. Soc. 2005, 26, 151-156.

13. Kim, S.; Jin, G.; Lim, M. J. Phys. Chem. B 2004, 108, 2036620375.

14. Hart, T. W. Tetrahedron Lett. 1985, 26, 2013-2016.

15. Mueller, R. P.; Huber, J. R. J. Phys. Chem. 1984, 88, 1605 1608.

16. Bartberger, M. D.; Houk, K. N.; Powell, S. C.; Mannion, J. D.; Lo, K. Y.; Stamler, J. S.; Toone, E. J. J. Am. Chem. Soc. 2000, 122, 5889-5890.

17. Picquart, M.; Grajcar, L.; Baron, M. H.; Abedinzadeh, Z. Biospectroscopy 1999, 5, 328-337.

18. Herzberg, G. Spectra of Diatomic Molecules; Van Nostrand Reinhold Ltd.: New York, 1950.

19. Wang, C.; Mohney, B. K.; Leecharoen, R.; Walker, G. C. Springer Series in Chemical Physics (Ultrafast Phenomena XI) 1998, 63, 603-605.

20. Lee, Y.-P., personal communication.

21. Kim, S.; Lim, M. J. Am. Chem. Soc. 2005, 127, 8908-8909.

22. Jackson, T. A. Ph.D. Thesis; Harvard University, Cambridge, 1996.

23. Lim, M.; Jackson, T. A.; Anfinrud, P. A. J. Chem. Phys. 1995 102, 4355-4366.

24. Sagnella, D. E.; Straub, J. E.; Jackson, T. A.; Lim, M.; Anfinrud, P. A. Proc. Natl. Acad. Sci. U.S.A. 1999, 96, 14324-14329.

25. Kennedy, G. R.; Ning, C. L.; Pfab, J. Chem. Phys. Lett. 1998, 292, 161-166.

26. Jimenez, R.; Flening, G. R.; Kumar, P. V.; Maroncelli, M. Nature 1994, 369, 471-473.

27. Miller, L. M.; Pedraza, A. J.; Chance, M. R. Biochemistry 1997, $36,12199-12207$. 